Scientific Visualization, 2019, volume 11, number 3, pages 126 - 132, DOI: 10.26583/sv.11.3.11

\title{
Method for visualization of macrodefects of dynamic periodic structures, based on the effect of the emergence of muar strips
}

\author{
N.T. Avlasevich¹, S.S. Anufrik², A.M. Lialikov³ \\ Yanka Kupala State University of Grodno \\ ${ }^{1}$ ORCID: 0000-0002-1525-0526, avlasevichnt@tut.by \\ 2 ORCID: 0000-0002-5761-4965, anufrick@grsu.by \\ 3 ORCID: 0000-0003-2525-6611, amlialikov@grsu.by
}

\begin{abstract}
$\underline{\text { Abstract }}$
Of particular interest are objects characterized by a tunable periodic structure. The structure parameters of such objects change not only in space, but also in time. The study of behavior in space and visualization of defects in periodic structures is one of the main tasks of measuring control.

The paper proposes a simple method of visualization of macrodefects of dynamic periodic structures based on the effect of the appearance of moire fringes. An easy-to-use visualization method involves registering a series of snapshots of a dynamic periodic structure followed by combining a selected pair of snapshots. The devices for recording a series of snapsohts and forming moiré paintings are described. A feature of the implementation of this method of visualization of macrodefects is the possibility of using incoherent light both when registering snapsohts and in the process of forming moire fringes. When creating a device for implementing the method, household LED lamps A60 with a matte bulb were used as light sources. A series of moire patterns was obtained, visualizing the spatial position of the macrodefect of the mask surface at various points in time of its mechanical deformation. A pattern of moire fringes, visualizing the position of a macrodefect of a periodic structure, was recorded by a digital camera and displayed on a personal computer monitor screen.
\end{abstract}

Keywords: dynamic periodic structure, macrodefect, moiré effect, a series of snapshots of the object, incoherent lighting, surface deformation.

\section{INTRODUCTION}

There are objects whose physical parameters of the structures are described by periodic functions. Such objects include diffractive elements of integrated and fiber optics, photonic crystal structures, transparent metamaterials, etc. [1-5]. The physical parameters of such objects change both in space and in time. Examples of such objects are photoinduced domain structures, electrically controlled liquid crystal elements, dynamic magnon crystals, etc. [6-8]. In addition to creating new technologies for manufacturing such structured objects, the task is to measure their parameters. One of the urgent tasks of quality control of structured objects is the identification, visualization and assessment of the macrodefects of a periodic structure.

The macroscopic defect of a periodic structure is understood to be the areas of the object in which the period of the structure deviates from a certain average value, as well as the deviation of the surface profile of the structure from a certain reference one. The dimensions of these defects significantly exceed the structure period. Marked macrodefects, in contrast to random formations, such as pollution or rupture of structural elements, can be determined by moiré, interference, and also shadow methods [9-12]. The implementation of these techniques is carried out with sources of coherent light for moiré and interference methods and non-coherent sources for moiré and shadow methods. However, it should be noted that the patterns that visualize the macrodefects of periodic structures obtained in incoherent light are of higher quality than the patterns obtained in coherent light. The impairment in the sec- 
ond case is due to the presence of speckle noise in images formed by coherent light. In this case, when using photosensitive arrays [13] for recording moiré patterns and subsequent digital processing, it is desirable to use incoherent light sources [14].

This article proposes an easy-to-technical method for visualizing macrodefects of a transmissive dynamic periodic structure using non-coherent lighting. This method consists of two stages. At the first stage, a series of snapshots of a dynamic periodic structure is recorded at various times during deformation surface. At the second stage of the method implementation, a picture of moiré fringes is observed on a matte screen background, when a selected pair of shots are combined, and the moiré patterns are displayed on a personal computer screen.

\section{REGISTRATION OF THE SERIES OF SNAPSHOTS}

Previously, the use of an incoherent light source in the visualization of macrodefects of periodic structures made it possible to obtain moiré and interference patterns of increased sensitivity with a sufficiently high quality [15-17]. However, in these works, the implementation of the method required the use of a reference snapshot of the periodic structure under study, and incoherent illumination was applied only at the final stage of the optical processing.

Let us consider the simplest case of a dynamic periodic structure, which is an object in the form of a thin amplitude transparency, the spatial transmission of which is described by a periodic function. In this case, we assume that the parameters describing the periodic structure of such an object change not only in space, but also in time.

In Fig. 1a shows the optical scheme explaining the registration of the $S$ snapshot of the studied dynamic periodic structure $O$. A series of shots is recorded in optically conjugated with the periodic structure $O$ under study, through a lens $L$ plane, in which the photographic material is set on a transparent basis. A diffuse scatterer $D$ was used to ensure uniform illumination of the periodic structure $O$, which was illuminated by a diverging incoherent light source $L s$. A film camera can be used as $L$ and $S$ elements.

We choose the directions of the axes plane coordinate system so that the y-axis was parallel to the strokes of the pattern of the periodic structure in the plane of the snapshot. In this case, the intensity distribution of light in the plane of the snapshot of the periodic structure under study can be represented as a Fourier series:

$$
I(x, y, t)=\sum_{n=0}^{+\infty} a_{n} \cos ^{2}\left[\frac{\pi x}{T}+\frac{1}{2} \varphi(x, y, t)\right]
$$

where $a_{n}$ are coefficients, $\mathrm{T}$ is a period of the structure, $\varphi(x, y, t)$ is a function that determines the distortions of the periodic structure and describes the macrodefect. The function can be represented as the sum of the individual components:

$$
\varphi(x, y, t)=\varphi_{1}(x, y, t)+\varphi_{2}(x, y, t),
$$

where $\varphi_{1}(x, y, t)$ is responsible for the violation of the periodicity of the elementary structure, due to the deviation of the period of strokes from a certain average value, and also because of the bending of strokes and contains all the information about the position of the stroke [18], $\varphi_{2}(x, y, t)$ characterizes the deviation of the surface shape of the structure from the plane and determines the change in the surface topography in space and time [19]. 


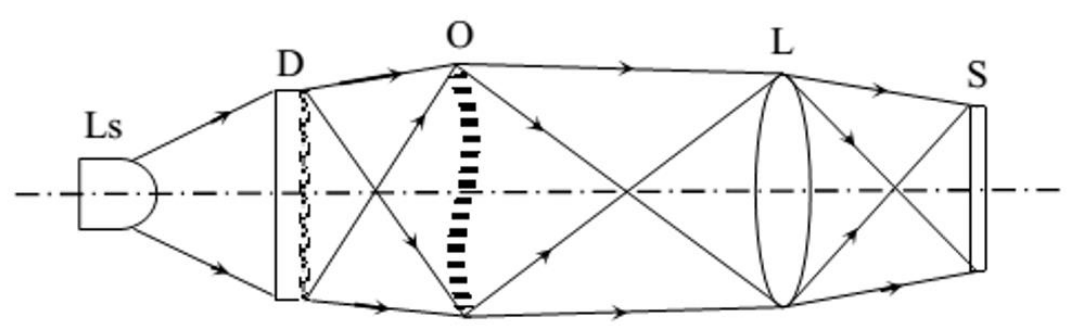

a)

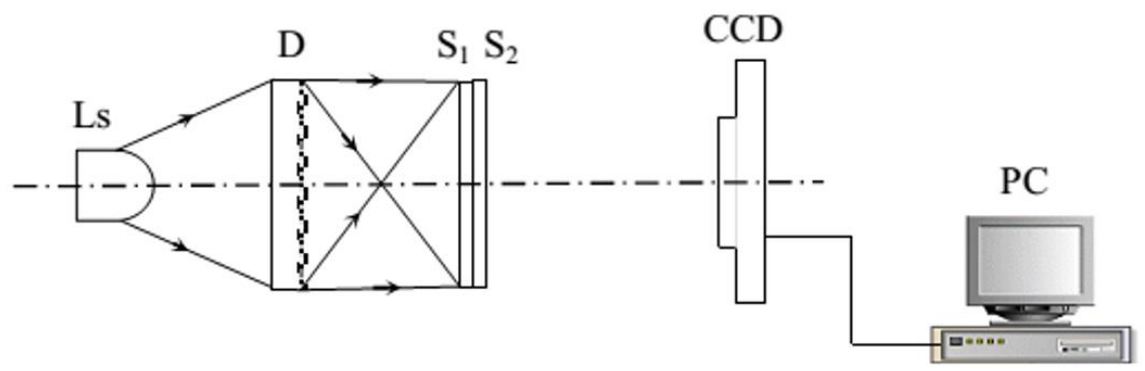

b)

Fig. 1. Optical schemes explaining: a - registration of the snapshot $S$ of the studied dynamic periodic structure, b - registration of the moiré pattern when combining a pair of snapshot $S_{1}$ and $S_{2}$ : Ls is the source of white light, $D$ is the diffuse scatterer, $O$ is the periodic structure under study, $L$ is the lens, $S$ - recorded snapshot, $S_{1}$ and $S_{2}$ - registered snapshots, $C C D$ - digital camera, $P C$ - personal computer.

Thus, against the background of the diffuse scatterer $D$, a series of snapshots $S$ of the periodic structure $O$ examined was recorded at different points in time. After chemical processing of the photographic material, the amplitude transmittances of the series of snapshots are $T_{1}\left(x, y, t_{1}\right), T_{2}\left(x, y, t_{2}\right), \ldots, T_{N}\left(x, y, t_{N}\right)$. Amplitude transmission coefficient of $k$-th snapshot is calculated using the formula:

$$
T_{k}\left(x, y, t_{k}\right)=\sum_{n=0}^{+\infty} b_{n} \cos \left[\frac{2 n \pi \alpha}{T}+n \varphi\left(x, y, t_{k}\right)\right],
$$

where $b_{n}$ are coefficients.

\section{VISUALIZATION OF THE MACRODEFECT DYNAMICS}

To determine the behavior of the dynamics of the function $\varphi(x, y, t)$, one can use optical methods based on the use of a reference periodic structure with a period close to the period $\mathrm{T}$ of the structure under study. Moire fringes that visualize the behavior of the function $\varphi(x, y$, $t$ ) in space and in time are formed when patterns of the studied and the reference periodic structures are superimposed [10]. In the absence of a reference periodic structure for the formation of a moiré pattern that visualizes macrodefects, one can use the overlay of two patterns of a periodic structure that are shifted relative to each other. Previously, this approach was used to determine the spatial position of surfaces with a periodic texture and also to identify macro and microdefects of amplitude masks $[19,20]$.

To determine changes in the function

$$
\Delta \varphi_{i k}(x, y)=\varphi_{i}\left(x, y, t_{l}\right)-\varphi_{k}\left(x, y, t_{k}\right),
$$

that occurred between the registration of two snapshots at times $t_{l}$ and $t_{k}$, the approach used in differential holographic interferometry can be used [21]. In differential holographic interferometry, the interference pattern is reconstructed using a hologram recorded on one medium by the method of two exposures at times $t_{l}$ and $t_{k}$, or two separate holograms recorded at corresponding times. However, in contrast to the differential interferometry method, the registration of snapshots of a dynamic periodic structure does not require optical recording schemes that are complex in the technical implementation, as well as optical processing. Fig. $1 \mathrm{~b}$ shows an optical scheme that allows forming a moiré pattern in incoherent light that re- 
flects the behavior of the function $\Delta \varphi_{l k}(x, y)$ during using two snapshots of a dynamic periodic structure with amplitude transmittances $T_{l}\left(x, y, t_{l}\right)$ and $T_{k}\left(x, y, t_{k}\right)$ recorded at times $t_{l}$ and $t_{k}$. The moiré pattern is observed when combining a pair of $S_{1}$ and $S_{2}$ snapshots against the background of the diffuser $D$ when the latter is illuminated with the light source Ls. When combining a pair of snapshots, the resulting amplitude transmittance $T_{l k}(x, y)$ is determined as $T_{l}\left(x, y, t_{l}\right)$ and $T_{k}\left(x, y, t_{k}\right)[22]$ or

$$
T_{i k}(x, y)=\sum_{n=0}^{+\infty} b_{n} \cos \left[\frac{2 n \pi x}{T}+n \varphi\left(x, y, t_{l}\right)\right] \sum_{n=0}^{+\infty} b_{n} \cos \left[\frac{2 n \pi x}{T}+n \varphi\left(x, y, t_{k}\right)\right] .
$$

The distribution of the light intensity $I_{l k}(x, y)$ at the output of the combined snapshots $S_{1}$ and $S_{2}$ will be proportional to the amplitude transmittance $T_{l k}(x, y)$, which is defined by the expression (5). To find the equation describing the middle of the moiré fringes corresponding to the regions with minimal visibility of the periodic structure, we restrict ourselves to the harmonics in expression (5), not higher than the main one. In this case

$$
\begin{aligned}
& I_{i k}(x, y)=\left\{c_{0}+c_{1} \cos \left[\frac{2 \pi x}{T}+\varphi\left(x, y, t_{l}\right)\right]\left\{c_{0}+c_{1} \cos \left[\frac{2 \pi x}{T}+\varphi\left(x, y, t_{k}\right)\right]\right\}=\right. \\
= & c_{0}^{2}+\frac{c_{l}^{2}}{2} \cos \left[\varphi\left(x, y, t_{l}\right)-\varphi\left(x, y, t_{k}\right)\right]+\frac{c_{l}^{2}}{2} \cos \left[\frac{4 \pi x}{T}+\varphi\left(x, y, t_{l}\right)+\varphi\left(x, y, t_{k}\right)\right]+ \\
+ & \frac{c_{0} c_{l}}{2} \cos \left\{\frac{l}{2}\left[\varphi\left(x, y, t_{l}\right)-\varphi\left(x, y, t_{k}\right)\right]\right\} \cos \left\{\frac{2 \pi x}{T}+\frac{I}{2}\left[\varphi\left(x, y, t_{l}\right)+\varphi\left(x, y, t_{k}\right)\right]\right\}
\end{aligned}
$$

where $c_{o}$ and $c_{1}$ are coefficients. The last term in expression (6) describes the moiré fringes corresponding to the areas of the combined snapshots in which the visibility of the periodic structure is minimal. Equating the last term in expression (6) to zero, we get the expression:

$$
\frac{c_{0} c_{1}}{2} \cos \left\{\frac{l}{2}\left[\varphi\left(x, y, t_{l}\right)-\varphi\left(x, y, t_{k}\right)\right]\right\} \cos \left\{\frac{2 \pi x}{T}+\frac{1}{2}\left[\varphi\left(x, y, t_{l}\right)+\varphi\left(x, y, t_{k}\right)\right]\right\}=0,
$$

taking into account the expression (5), we obtain the equations describing the middle of the moiré fringes:

where $m=0,1,2, \ldots$

$$
\varphi_{i k}(x, y)=\pi(2 m+I),
$$

Thus, moiré fringes correspond to areas of the pattern in which the visibility of a periodic structure with a period $T$ takes on a minimum value.

\section{EXPERIMENT}

To confirm the operability of the visualization method of macrodefects of dynamic periodic structures, a periodic metal mask was chosen as the object under study, the surface of which was subjected to mechanical deformation. When the surface of the metal mask was deformed, its surface deviated from the plane. A series of snapshots of this object was recorded with Zenit TTL film mirror camera on Micrat 300 film. The developer was Kodak D-19 contrast developer which provides a high-contrast image of the periodic structure under study.

The restoration of the moiré patterns which reflect the dynamics of the development of the deformation of the surface of the metal mask under investigation using a pair of snapshots recorded at different points in time was carried out in the scheme shown in Fig. 1b. Moiré patterns were recorded with a $C C D$ digital camera while focusing on the plane of the combined $S_{1}$ and $S_{2}$ snapshots set against the diffuse scatterer $D$. It should be noted that during creating the device layouts shown in Fig. 1a and Fig. 1b, a common lighting system was used. A LED A6o lamp with a $7 \mathrm{~W}$ matt bulb was used as the Ls light source. The combination of snapshots $S_{1}$ and $S_{2}$ was carried out in a special optical device designed to combine holograms. This device provided an absolute error of relative shift of images at the level of $5 \mathrm{mi}$ crons [21]. 
In Fig. 2 shows photographs of moiré patterns obtained in the scheme (Fig. 1b) when using pairs of snapshots recorded at different points in time $t_{1}=1 \mathrm{~s}, t_{2}=3 \mathrm{~s}, t_{3}=5 \mathrm{~s}, t_{4}=8 \mathrm{~s}$ and $t_{5}=12 \mathrm{~s}$ after the onset of deformation of the metal surface masks.

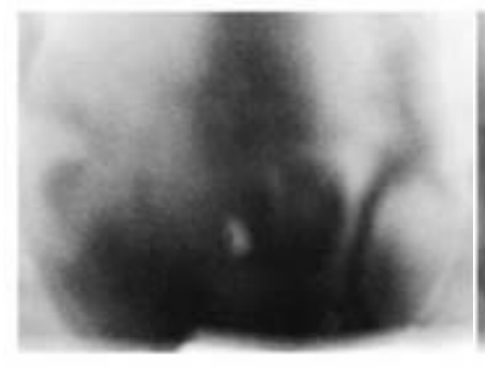

a)

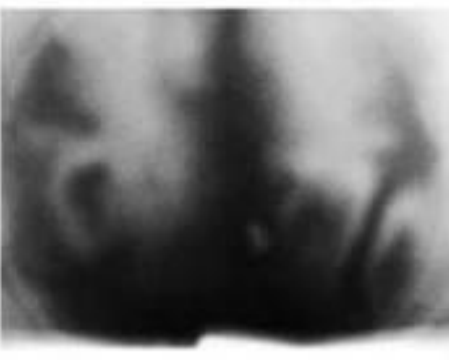

b)

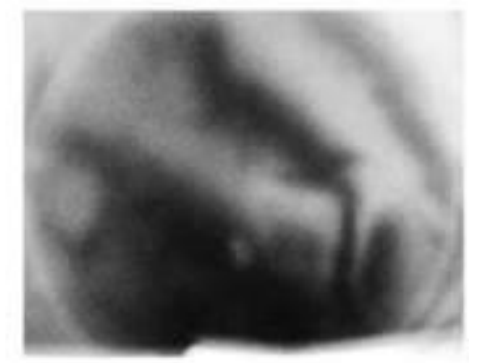

c)

Fig. 2. Photos of moiré patterns of pairs of snapshots recorded at different points in time: a) $t_{1}$ and $\left.t_{3}, \mathrm{~b}\right) t_{3}$ and $\left.t_{5}, \mathrm{c}\right) t_{2}$ and $t_{4}$

Moiré patterns show changes in the surface profile of a metal mask that occurred due to the deformation of its surface over time intervals, corresponding to the registration of pairs of snapshots with amplitude transmittances: $T_{1}\left(x, y, t_{1}\right)$ and $T_{3}\left(x, y, t_{3}\right)$ (Fig. 2a ); $T_{3}\left(x, y, t_{3}\right)$ and $T_{5}\left(x, y, t_{5}\right)$ (Fig. 2b); $T_{2}\left(x, y, t_{2}\right)$ and $T_{4}\left(x, y, t_{4}\right)$ (Fig. 2c).

\section{CONCLUSION}

A relatively simple from the point of view of technical implementation method of visualizing macrodefects of a transient dynamic periodic structure is theoretically substantiated and experimentally confirmed. The method is based on the effect of the formation of moiré fringes when snapshots of the studied structure recorded at different points in time are combined. Affordable equipment is used for prototyping of devices. To obtain quantitative information moiré patterns can be processed using known algorithms for the digital processing of interferograms [23].

\section{References}

1. Liu N. [et al.] Three-dimensional photonic metamaterials at optical frequencies. Nature Materials, 2008, vol. 7, pp. 31-37.

2. Denisyuk I. Yu. [et al.] An optical narrow-band filter based on a Bragg grating recorded in a microchannel waveguide filled with a photopolymer. Optical Technology, 2013, vol. 8o, pp. 193-196.

3. Liao C. R., Wang D. N. Review of femtosecond laser fabricated fiber Bragg gratings for high temperature sensing. Photonic Sensors, 2013, vol. 3, No 2, pp. 97-101.

4. Viegas D. Long-Period Grating Fiber Sensor With In Situ Optical Source for Remote Sensing. Photonics Technology Letters, 2010, vol. 22, pp. 1533-1535.

5. Vendik I.B., Vendik O.G. Metamaterials and their application in microwaves: a review. Journal of Technical Physics, 2013, vol. 58, No 1, pp. 1-24.

6. Ustinova I. A., Nikitin A. A., Ustinov A. B. Dynamic magnonic crystal based on a layered ferrite-ferroelectric structure. Technical Physics, 2016, vol. 61, No 3, pp. 473-476.

7. Kabanova O. S. [et al.] Electrically controlled waveguide liquid-crystal elements. Technical Physics Letters, 2014, vol. 40, No 7, pp. 598-600.

8. Golenishchev-Kutuzov A. V. [et. al] Tunable acoustic resonator on a periodic domain structure. Technical Physics Letters, 2012, vol. 38, No 9, pp. 825-827.

9. Groshenko N. A., Makalish O. S., Volyar A. V. Optical vortices in the scattering field of magnetic domain holograms. Technical Physics, 1998, vol. 43, No 12, pp. 1450-1453.

10. Durelli A.J., Parks V.J. Moiré analysis of strain [Analiz deformacij s ispol'zovaniem muara]. Moscow, 1974, 353 p. [in Russian] 
11. Lyalikov A. M. Visualizing macroscopic surface defects of an object with a periodic structure, Optical Technology, 1995, vol. 62, No 1, pp. 21-23.

12. Avlasevich N. T., Lyalikov A. M. Visualization of the defects of a separate component of a composite diffractive optical element. PFMT (Problemy Fiziki, Matematiki i Tekhniki (Problems of Physics, Mathematics and Technics)), 2017, No. 3(32), pp. 7-12. [in Russian]

13. Gusev M.E. [et. al] Methods of digital holographic interferometry and its application to measure nanodisplacement [Metody cifrovoj golograficheskoj interferometrii i ih primenenie dlya izmereniya nanoperemeshchenij] Nanosystems: Physics, Chemistry, Mathematics, 2011, vol. 2, No 1, pp. 23-39. [in Russian]

14. Schnars U., Jueptner W. Digital Holography. Berlin, 2004, 164 p.

15 Lyalikov A. M. Enhancement of the measurement sensitivity in real-time optical image processing for objects with a periodic structure. Technical Physics, 1999, vol. 44, No 7, pp. 862-863.

16. Lyalikov A. M. High-sensitivity moiré flaw detection for comparison of periodic composite structures. Technical Physics, 2001, vol. 46, No 5, pp. 587-589.

17. Dich L. Z. On the Accuracy of Diffractometric Certification of Periodic Standards. Optics and Spectroscopy, 1997, vol. 83, No. 3, pp. 477-482.

18. Lyalikov A. M. Visualization of the surface shape of objects with periodic structure. Journal of Optical Technology, 1994, vol. 61, No 5, pp. 376-378.

19. Lyalikov A. M. Double-exposure holographic recording of information in studies of the macrorelief of diffusely scattering surfaces by fringe projection method. Optics and Spectroscopy, 1996, vol.8o, pp. 765-772.

20. Lyalikov A. M. Revealing macrodefects in periodic structures of the transmission type in white light on the basis of shift of images. Optics and Spectroscopy, 2005, vol. 98, No 3, pp. 477-482.

21. Beketova A. K. [et al.]; ed. Mishina G. I. Holographic interferometry of phase objects [Golograficheskaia interferometriia fazovykh ob'ektov ]. Leningrad, 1979, 232 p. [in Russian]

22. Vest Ch. Holographic interferometry [Golograficheskaia interferometriia]. Moscow, 1982, 504 p. [in Russian]

23. Malacara D., Servín M., Malacara Z. Interferogram Analysis for Optical Testing. London, 2005, $568 \mathrm{p}$. 